\title{
Formation history of open clusters constrained by detailed asteroseismology of red giant stars observed by Kepler
}

\author{
Enrico Corsaro ${ }^{1,2,3, \star}$, Yueh-Ning Lee ${ }^{1}$, Rafael A. García ${ }^{1}$, Patrick Hennebelle ${ }^{1}$, Savita Mathur ${ }^{4}$, Paul G. Beck ${ }^{1}$, \\ Stephane Mathis ${ }^{1}$, Dennis Stello ${ }^{5,6}$, and Jérôme Bouvier ${ }^{7}$ \\ ${ }^{1}$ Laboratoire AIM Paris-Saclay, CEA/DRF — CNRS — Université Paris Diderot, IRFU/SAp Centre de Saclay, F-91191 Gif-sur- \\ Yvette Cedex, France \\ ${ }^{2}$ Instituto de Astrofísica de Canarias, E-38200 La Laguna, Tenerife, Spain \\ ${ }^{3}$ Departamento de Astrofísica, Universidad de La Laguna, E-38205 La Laguna, Tenerife, Spain \\ ${ }^{4}$ Space Science Institute, 4750 Walnut street Suite 205, Boulder, CO 80301, USA \\ ${ }^{5}$ Sydney Institute for Astronomy (SIfA), School of Physics, University of Sydney, Sydney, New South Wales 2006, Australia \\ ${ }^{6}$ Stellar Astrophysics Centre, Department of Physics and Astronomy, Aarhus University, Ny Munkegade 120, DK-8000 Aarhus C, \\ Denmark \\ ${ }^{7}$ Université Grenoble Alpes, IPAG, F-38000 Grenoble, France; CNRS, IPAG, F-38000 Grenoble, France
}

\begin{abstract}
Stars originate by the gravitational collapse of a turbulent molecular cloud of a diffuse medium, and are often observed to form clusters. Stellar clusters therefore play an important role in our understanding of star formation and of the dynamical processes at play. However, investigating the cluster formation is difficult because the density of the molecular cloud undergoes a change of many orders of magnitude. Hierarchical-step approaches to decompose the problem into different stages are therefore required, as well as reliable assumptions on the initial conditions in the clouds. We report for the first time the use of the full potential of NASA Kepler asteroseismic observations coupled with 3D numerical simulations, to put strong constraints on the early formation stages of open clusters. Thanks to a Bayesian peak bagging analysis of about 50 red giant members of NGC 6791 and NGC 6819, the two most populated open clusters observed in the nominal Kepler mission, we derive a complete set of detailed oscillation mode properties for each star, with thousands of oscillation modes characterized. We therefore show how these asteroseismic properties lead us to a discovery about the rotation history of stellar clusters. Finally, our observational findings will be compared with hydrodynamical simulations for stellar cluster formation to constrain the physical processes of turbulence, rotation, and magnetic fields that are in action during the collapse of the progenitor cloud into a proto-cluster.
\end{abstract}

\section{Introduction}

Most of the star formation in the Galaxy is occurring within massive giant molecular clouds [1]. Giant molecular clouds are expected to form hundred of thousands of stars that can originate stellar clusters if the system is able to remain gravitationally bound well beyond its formation phase. This shows that investigating the early dynamical evolution of the molecular clouds is crucial for understanding the star and planet formation processes at different epochs and by taking into account the diversity of environments in the Galaxy [2,3]. However, the star forming regions are obscured by dust, hence direct observations are limited to the infrared and radio bands. Stellar clusters, which are born embedded to giant molecular clouds, therefore represent important benchmarks to test stellar and planetary formation, and stellar evolution theories. Open clusters in particular generally contain a small amount or no interstellar medium, which makes them well suitable for studies involving a broad range of wavelengths other

\footnotetext{
^e-mail: enrico.corsaro@cea.fr
}

than the infrared and radio ones. In addition, conversely to globular clusters, stars in open clusters are sparse enough, with a density of at least $1 M_{\odot} \mathrm{pc}^{-3}$, thus providing the opportunity to perform detailed analyses on individual stellar members. The great advantage of this approach is that the stars in a cluster - as opposed to field stars that often originate from the dissolution of small stellar systems can preserve the signature of the initial conditions of the progenitor cloud.

Little is known on the role of angular momentum on the early stage formation of stars in open clusters. It is believed that molecular clouds satisfying the Jeans instability undergo a gravitational collapse in which the internal motions are strongly influenced by turbulence [4]. This implies that the angular momentum from the progenitor gas cloud cannot leave any significant imprint of their action in the resulting stars of the cluster. From a theoretical point of view, if the angular momentum vector of the cloud is transferred to the individual pre-stellar cores then rotation and possibly magnetic fields could be responsible of guiding the gravitational accretion of mass along preferential 
directions in space. For understanding this aspect, reliable measurements of the space orientation of the stellar-spin axis are required. Previous analyses conducted on young open clusters support no evidence of stellar-spin alignment [5], but they relied on a combination of measurements for stellar rotational periods from light curves, projected equatorial velocities from spectroscopy, and stellar radii based on the cluster distance. As a result, the adopted methodology could be prone to significant systematics, and it is not applicable for distant and old populations of stars, whereas the required primary information will lack.

Asteroseismology, the study of stellar oscillations, has proven to be a powerful tool to extract direct information on the stellar interiors, and in particular on the stellar angular momentum vector, thus allowing for accurate and detailed results, especially in the case of red giant stars [6]. Red giants are low- and intermediate-mass stars that have evolved off the main sequence to settle in either a hydrogen-shell-burning or helium-core-burning phase of the stellar evolution. Many red giants exhibit stellar oscillations that can be analyzed through a Fourier spectrum of the light curve. Such a Fourier spectrum contains a regular pattern of tens and sometimes more than a hundred of radial and non-radial stellar oscillation modes [7]. Each oscillation mode can be identified by means of an angular degree $l$ and an azimuthal number $m$, the latter related to the degeneracy lifted by the stellar rotation to form a multiplet of $(2 l+1)$ components [8,9]. The dipolar $(l=1)$ mixed modes [10] are the most suited for measuring the orientation of the spin axis.

\section{Data analysis \& results}

Here we investigate 48 oscillating red giant members of the open clusters NGC 6791 and NGC 6819 [11-13], with masses spanning from 1.1 to $1.7 M_{\odot}$ [14]. Both clusters are old, with NGC 6791 one of the oldest known in our Galaxy [15]. Despite their distance (on galactic scales up to $\sim 4 \mathrm{kpc}$ ), thanks to the high luminosity of the red giants, the individual stellar members could be monitored by the photometric space mission NASA Kepler for a period of about 4 years. By performing detailed asteroseismic analyses on each star [16-20], we obtain a total of about 380 rotationally split dipolar mixed modes - identified from a set of more than 3900 oscillation modes — and we use them to measure the spin inclination angles.

The analysis of the rotationally split dipolar mixed modes shows that about $70 \%$ of the stars in each cluster has a strong preferential spin orientation, with inclination angles between $20^{\circ}$ and $30^{\circ}$. This is contrary to what would be expected by assuming an uniform 3D distribution of spin vectors, where most of the inclination angles would then be found close to $90^{\circ}$. The spatial distribution of the stars also guarantees that they adequately sample the observed spherical morphology of the two stellar clusters.

Existing N-body simulations for old open clusters [21] show that the stellar angular momentum can influence the evolution of colliding stars and of the orbital configurations of multiple stellar systems, e.g. the orbital eccentricities and periods of binaries. In the case of single stars we instead observe only a spin down over time [22]. Because of the larger average distances among the star members of an open cluster, the N-body interactions cannot produce any appreciable effect on redistributing stellar spin inclinations and yielding a spin alignment even for timescales of several Gyr. As a consequence, the spin alignment that is observed in the stars of our sample should have a different origin, linked to the formation epoch of the cluster.

We have performed $3 \mathrm{D}$ hydrodynamical simulations that reproduce the collapse of a dense molecular cloud forming a proto-cluster [23]. The simulations show that when we consider a cloud dominated by turbulent kinetic energy, $E_{\text {tur }}$, over that of global rotation, $E_{\text {rot }}$ (yielding the condition $E_{\mathrm{rot}} / E_{\mathrm{tur}}<1$ ), no significant stellar spin alignment is produced in the final pre-stellar cores and the distribution of spin inclinations resembles that of a uniform orientation in 3D space. The scenario becomes comparable to that of our observations when $E_{\text {rot }} / E_{\text {tur }} \simeq 1$ instead. In addition, when considering masses below $0.7 M_{\odot}$, even an energy equipartition between rotational and turbulent kinetic energy is not able to produce a significant spin alignment in the resulting pre-stellar cores.

\section{Conclusion}

Our results indicate that a mass above $0.7 M_{\odot}$, accreted into a pre-stellar core from the progenitor molecular cloud, would be required in order to allow the cloud's average angular momentum to be transferred from the scale of the molecular cloud to those of individual stars. At least $50 \%$ of the cloud's rotational kinetic energy can be responsible of efficiently aligning the spin axes within the stellar members of a cluster. Therefore, the two open clusters NGC 6791 and NGC 6819 are likely to be originating from a formation process that involved a dense collapsing molecular cloud with a strong rotational energy component. Through the measurement of stellar spin inclinations for stars with masses comparable to that of our Sun and belonging to open clusters we can obtain information on the initial energy budget of the progenitor molecular cloud, its global rotation, and the efficiency by which the cloud's averaged angular momentum is transferred to the individual stellar members of the clusters. This will lead to the exploration and reconstruction of the dynamical evolution of galactic star forming regions that have originated clusters with ages up to several Gyr.

\section{Acknowledgements}

E.C. is funded by the European Community's Seventh Framework Programme (FP7/2007-2013) under grant agreement $\mathrm{n}^{\circ} 312844$ (SPACEINN). Y.-N.L. and P.H. acknowledge funding by the European Research Council under the European Community's Seventh Framework Programme (FP7/2007-2013 Grant Agreement no. 306483) and the HPC resources of CINES under the allocation x2014047023 made by GENCI (Grand Equipement National de Calcul Intensif). R.A.G. received funding from the CNES GOLF and PLATO grants at CEA. R.A.G. and P.G.B. received funding from the ANR (Agence Nationale de 
la Recherche, France) program IDEE (n ANR-12-BS05-0008) "Interaction Des Étoiles et des Exoplanètes". Sa.M. acknowledges support from the NASA grant NNX12AE17G. St.M. acknowledges funding by the European Research Council through ERC grant SPIRE 647383. D.S. is the recipient of an Australian Research Council Future Fellowship (project number FT140100147). J.B. acknowledges financial support from grant ANR 2011 Blanc SIMI5-6 020 "Toupies: Towards understanding the spin evolution of stars". This work has received funding from the CNES grants at CEA.

\section{References}

[1] Lee, E. J., Murray, N., \& Rahman, M. 2012, ApJ, 752,146

[2] Lada, C. J. \& Lada, E. A. 2003, ARA\&A, 41, 57

[3] Longmore, S. N., Kruijssen, J. M. D., Bastian, N., et al. 2014, Protostars and Planets VI, 291

[4] Shu, F. H., Adams, F. C., \& Lizano, S. 1987, ARA\&A, 25, 23

[5] Jackson, R. J. \& Jeffries, R. D. 2010, MNRAS, 402, 1380

[6] Huber, D., Carter, J. A., Barbieri, M., et al. 2013, Science, 342, 331

[7] Bedding, T. R., Mosser, B., Huber, D., et al. 2011, Nature, 471, 608

[8] Beck, P. G., Montalban, J., Kallinger, T., et al. 2012, Nature, 481, 55
[9] Gizon, L. \& Solanki, S. K. 2003, ApJ, 589, 1009

[10] Beck, P. G., Bedding, T. R., Mosser, B., et al. 2011, Science, 332, 205

[11] Basu, S., Grundahl, F., Stello, D., et al. 2011, ApJ, 729, L10

[12] Corsaro, E., Stello, D., Huber, D., et al. 2012, ApJ, 757, 190

[13] Stello, D., Meibom, S., Gilliland, R. L., et al. 2011, ApJ, 739, 13

[14] Miglio, A., Brogaard, K., Stello, D., et al. 2012, MNRAS, 419, 2077

[15] Brogaard, K., VandenBerg, D. A., Bruntt, H., et al. 2012, A\&A, 543, A106

[16] García, R. A., Hekker, S., Stello, D., et al. 2011, MNRAS, 414, L6

[17] García, R. A., Mathur, S., Pires, S., et al. 2014, A\&A, 568, A10

[18] Mathur, S., García, R. A., Régulo, C., et al. 2010, A\&A, 511, A46

[19] Corsaro, E. \& De Ridder, J. 2014, A\&A, 571, A71

[20] Corsaro, E., De Ridder, J., \& García, R. A. 2015, A\&A, 579, A83

[21] Geller, A. M., Hurley, J. R., \& Mathieu, R. D. 2013, AJ, 145, 8

[22] Meibom, S., Barnes, S. A., Platais, I., et al. 2015, Nature, 517, 589

[23] Lee, Y.-N. \& Hennebelle, P. 2016, A\&A, 591, A30 